\title{
Solução Numérica para um Problema de Cauchy Fuzzy que Modela o Decaimento Radioativo
}

G.L. DINIZ1, Departamento Matemática, ICET, UFMT, 78.060-900 Cuiabá, MT, Brasil

J.F.C.A. MEYER ${ }^{2}$, L.C. BARROS, Departamento de Matemática Aplicada, IMECC, UNICAMP, 13.083-970 Campinas, SP, Brasil.

\begin{abstract}
Resumo. O uso de materiais radioativos no último século trouxe sérios riscos para o meio ambiente, principalmente, com relação aos depósitos de lixo radioativo. Neste artigo, propomos um modelo fuzzy para descrever o fenômeno de decaimento radioativo, usando equações diferenciais fuzzy, cuja solução analítica clássica é bem conhecida. Aplicamos métodos do tipo Euler e Runge-Kutta para obter uma solução aproximada de um problema de valor inicial da equação diferencial ordinária linear fuzzy, que modela o decaimento radioativo. Os resultados numéricos fuzzy obtidos são comparados com a solução analítica fuzzy apresentada por Barros et al [2] para uma equação diferencial fuzzy similar.
\end{abstract}

\section{Introdução}

Segundo Ma, Friedman e Kandel [10], Chang e Zadeh [4] foram os primeiros a introduzir o conceito de derivadas fuzzy. Mais tarde, Dubois e Prade [5] usaram o princípio de extensão e suas aproximações, para obter os primeiros resultados com o cálculo diferencial para função fuzzy. Puri e Ralescu [13] discutiram outros métodos onde propuseram duas definições, uma baseada na diferencial de Hukuhara de uma multifunção, limitada ao cone convexo, e outra para espaços de Banach (ver [10]).

Kandel e Byatt [8, 9] aplicaram o conceito de equações diferenciais fuzzy para problemas dinâmicos fuzzy, mas foram Kaleva, Nieto, Ouyang et al e Seikkala [6, 7, $11,12,14]$ quem deram um tratamento rigoroso ao problema de valor inicial fuzzy (problema fuzzy de Cauchy).

Wu, Song e Lee [15] obtiveram um teorema de existência e unicidade de solução do problema de Cauchy para as equações diferenciais fuzzy: $x^{\prime}(t)=f(t, x(t))$, $x\left(t_{0}\right)=x_{0}$ para aplicações fuzzy de variável real, cujos conjuntos são normais, convexos e de suporte compacto em $\mathbb{R}^{n}$ com a função $f$ satisfazendo a condição generalizada de Lipchitz.

\footnotetext{
${ }^{1}$ dinizgl@densis.fee.unicamp.br

2 joni@ime.unicamp.br

${ }^{3}$ laeciocb@ime.unicamp.br
} 
A solução analítica fuzzy do problema de Cauchy para equação diferencial fuzzy de modelos que descrevem a dinâmica populacional foi apresentada em Barros et al [2].

A resolução de equações diferenciais fuzzy, via aproximação numérica, também é recente e uma das poucas referências é Ma, Friedman e Kandel [10], onde é apresentado um algoritmo numérico para resolver uma equação diferencial ordinária fuzzy, com um esquema baseado no clássico método de Euler, seguido de uma análise de erro e ilustrado com alguns problemas de Cauchy (linear e não-linear).

\section{Preliminares}

Seja $\mathcal{U}$ um conjunto (no sentido clássico). Um subconjunto fuzzy $\mathbb{F}$ em $\mathcal{U}$ é o conjunto $\mathbb{F}=\{(x, \mu(x))\}$, onde $\mu: \mathcal{U} \longrightarrow[0,1]$ é a função grau de pertinência de $x \in \mathcal{U}$ ao conjunto fuzzy $\mathbb{F}$. Assim, o subconjunto fuzzy $\mathbb{F}$ é definido por sua função $\mu \mathrm{e}$, muitas vezes, indicado por $\mu_{\mathbb{F}}$. Esta função pode ser pensada como uma generalização da função característica de um conjunto clássico.

Considere $\mathbb{E}=\{\mu: \mathbb{R} \longrightarrow[0,1]\}$, onde $\mu$ satisfaz:

i. $\exists x_{0} \in \mathbb{R}$ tal que $\mu\left(x_{0}\right)=1$;

ii. $\forall \mathrm{x}, \mathrm{y} \in \mathbb{R}, \mu(\delta x+(1-\delta) y) \geq \min \{\mu(x), \mu(y)\}, \forall \delta \in[0,1]$;

iii. $\mu$ é semicontínua superiormente;

iv. $\overline{\{x \in \mathbb{R} \mid \mu(x)>0\}}$ é compacto.

Neste caso, costuma-se dizer que o conjunto fuzzy é um número fuzzy.

Se $\mu \in \mathbb{E}$, definimos o nível $\alpha$ de $\mu$ por:

$$
[\mu]^{\alpha}=\{x \in \mathbb{R} \mid \mu(x) \geq \alpha\}, 0<\alpha \leq 1 .
$$

Assim, temos que $[\mu]^{\alpha}$ é um intervalo fechado $\left[\mu_{1}^{\alpha} ; \mu_{2}^{\alpha}\right]$ para todo $\alpha$ com

$$
0<\alpha \leq 1, \mathrm{e}[\mu]^{0}=\overline{\{x \in \mathbb{R} \mid \mu(x)>0\}} .
$$

Temos, ainda, que se $\mu, \nu \in \mathbb{E}$, então $\mu=\nu \Longleftrightarrow[\mu]^{\alpha}=[\nu]^{\alpha}, \forall \alpha \in(0,1]$.

Teorema 1 (de Representação [6, 14]) Seja $\left[\mu_{1}^{\alpha}, \mu_{2}^{\alpha}\right]$ uma família de intervalos não vazios, $0<\alpha \leq 1$. Se

i. $\left[\mu_{1}^{\alpha_{i}}, \mu_{2}^{\alpha_{i}}\right] \subset\left[\mu_{1}^{\alpha_{j}}, \mu_{2}^{\alpha_{j}}\right]$ para $0<\alpha_{j} \leq \alpha_{i} i, j=1$, 2 e

ii. $\left[\lim _{k \rightarrow \infty} \mu_{1}^{\alpha_{k}} ; \lim _{k \rightarrow \infty} \mu_{2}^{\alpha_{k}}\right]=\left[\mu_{1}^{\gamma} ; \mu_{2}^{\gamma}\right] \operatorname{com} \alpha_{k} \uparrow \gamma$ e $\gamma \in(0,1]$;

então, $\exists \mu \in \mathbb{E} \operatorname{com}[\mu]^{\alpha}=\left[\mu_{1}^{\alpha}, \mu_{2}^{\alpha}\right], \forall \alpha \in(0,1] e[\mu]^{0}=\overline{\bigcup_{0<\alpha \leq 1}\left[\mu_{1}^{\alpha} ; \mu_{2}^{\alpha}\right]}$. 
Inversamente, se $\mu \in \mathbb{E}$, então $[\mu]^{\alpha} \operatorname{satisfaz~(i)~e~(ii).~}$

Em virtude do Teorema de Representação podemos definir operações entre números fuzzy através de seus $\alpha$-níveis.

Sejam $\mu$ e $\nu$ elementos de $\mathbb{E}$, com $[\mu]^{\alpha}=\left[\mu_{1}^{\alpha}, \mu_{2}^{\alpha}\right]$ e $[\nu]^{\alpha}=\left[\nu_{1}^{\alpha}, \nu_{2}^{\alpha}\right]$, então:

(a) $\mu+\nu=[\mu+\nu]^{\alpha} \doteq\left[\mu_{1}^{\alpha}+\nu_{1}^{\alpha} ; \mu_{2}^{\alpha}+\nu_{2}^{\alpha}\right]$; (adição fuzzy).

(b) $\mu \times \nu \doteq\left[\min \left\{\mu_{i}^{\alpha} ; \nu_{j}^{\alpha}\right\} ; \max \left\{\mu_{i}^{\alpha} ; \nu_{j}^{\alpha}\right\}\right] ; \mathrm{i}, \mathrm{j}=1,2 ;$ (multiplicação fuzzy).

\section{Descrição do Problema e o Modelo Matemático}

O número de desintegrações por unidade de tempo mede a atividade radioativa de uma substância. Este fenômeno é devido à emissão de três tipos de radiações: partículas $\alpha$ (núcleos de hélio), partículas $\beta$ (elétrons) e raios $\gamma$ (ondas eletromagnéticas de alta freqüência). Os primeiros experimentos que resultaram em tal compreensão foram realizados por Rutheford, Becquerel, Royds, Vilard e M. Curie no final do século XIX e início do XX, quando já se sabia que a atividade é proporcional ao número de átomos radioativos presentes em cada instante.

O uso desse tipo de material deixa o público em geral em estado de alerta, e os benefícios que o uso de tais materiais pode trazer, somente podem ser avaliados se os seus perigos são dimensionados. O primeiro passo desse processo seria, talvez, o conhecimento das origens desses materiais.

A formulação matemática (ver Bassanezzi e Ferreira [3]) que modela o processo de decaimento radioativo, pode ser descrita da seguinte forma:

$$
\frac{d N}{d t}=-\lambda N
$$

onde $N=N(t)$ é o número de átomos radioativos presentes na amostra no instante $\mathrm{t}$ e $\lambda>0$ é a constante de desintegração radioativa, que pode ser obtida experimentalmente.

O sinal negativo na equação (3.1) é porque o número de átomos radioativos diminui com o passar do tempo, com isso a taxa de variação instantânea $d N / d t$ deve ser negativa. A constante de desintegração $\lambda$, característica de cada elemento radioativo, permite dizer se este elemento tem vida curta ou longa.

A solução analítica clássica da equação (3.1) é dada por:

$$
N(t)=N_{0} e^{-\lambda t},
$$

sendo $N_{0}=N(0)$ a quantidade inicial destes átomos.

Levando em conta que $N=\left(N_{a} / A\right) m$, onde A é o número de massa do elemento radioativo e $N_{a}$ é o número de Avogadro, que vale $6,02 \times 10^{23} \mathrm{mols}^{-1}$, daí a razão $N_{a} / A$ é constante para cada elemento.

Assim, em termos da massa do material radioativo, a lei de atividade pode ser expressa por:

$$
m(t)=m_{0} e^{-\lambda t} .
$$


Este é o tratamento clássico para o problema, no entanto, apesar da simplicidade da equação a dificuldade maior está na contagem, para a obtenção dos parâmetros identificados no modelo. Os dados coletados, bem como o método adotado, quase sempre estão carregados de imprecisão e elementos de incerteza causados tanto pelo próprio processo de medição, como por algum tipo de subjetividade na adoção do método.

Desta forma, com o intuito de dar um tratamento matemático às questões que apresentam incertezas, o uso de sistemas fuzzy pode ser visto como uma ferramenta indispensável para a análise e compreensão do fenômeno em estudo. Para isto, adotaremos o processo "fuzzyness" como em Barros et al [1] e Kaleva [6].

Para uma primeira aproximação, considerando a condição inicial como um conjunto fuzzy, adotaremos a seguinte equação diferencial ordinária fuzzy:

$$
u^{\prime}(t)=-\lambda u(t), \quad u(0)=u_{0} \in \mathbb{E},
$$

onde $u^{\prime}(t)$ é a derivada de Hukuhara, $u: \mathbb{R}_{+} \longrightarrow \mathbb{E}$, e $t, \lambda \in \mathbb{R}_{+}$, ou seja,

$$
[u]^{\alpha}=\left[\left[u_{1}\right]^{\alpha} ;\left[u_{2}\right]^{\alpha}\right] .
$$

A existência e unicidade da solução de (3.4), para $\mathrm{T}$ intervalo fechado de $\mathbb{R}_{+}$, é apresentada em Barros et al, Kaleva e Nieto [1, 6] e [11].

Em virtude do Teorema de representação e levando em conta as operações definidas por (a) e (b), o problema de Cauchy fuzzy: $u^{\prime}(t)=-\lambda u(t), u(0)=u_{0}$, é equivalente à família de sistemas bidimensionais clássicos:

$$
\left\{\begin{array}{lll}
{\left[u_{1}^{\prime}(t)\right]^{\alpha}=-\lambda\left[u_{2}(t)\right]^{\alpha},} & {\left[u_{1}(0)\right]^{\alpha}=\left[u_{1_{0}}\right]^{\alpha},} & 0<\alpha \leq 1, \\
{\left[u_{2}^{\prime}(t)\right]^{\alpha}=-\lambda\left[u_{1}(t)\right]^{\alpha},} & {\left[u_{2}(0)\right]^{\alpha}=\left[u_{2_{0}}\right]^{\alpha},} & 0<\alpha \leq 1,
\end{array}\right.
$$

onde $\left[u_{1}(t)\right]^{\alpha}$ e $\left[u_{2}(t)\right]^{\alpha}$ são os $\alpha$-níveis da solução em cada instante t, $\left[u_{1_{0}}\right]^{\alpha}$ e $\left[u_{2_{0}}\right]^{\alpha}$ são os $\alpha$-níveis de $u_{0}$.

Para cada $\alpha$, a solução analítica do sistema descrito por (3.5), conforme Barros et al [2], é dada por:

$$
\left\{\begin{array}{l}
{\left[u_{1}(t)\right]^{\alpha}=\frac{\left[u_{1_{0}}\right]^{\alpha}-\left[u_{2_{0}}\right]^{\alpha}}{2} e^{\lambda t}+\frac{\left[u_{1_{0}}\right]^{\alpha}+\left[u_{2_{0}}\right]^{\alpha}}{2} e^{-\lambda t},} \\
{\left[u_{2}(t)\right]^{\alpha}=\frac{\left[u_{2_{0}}\right]^{\alpha}-\left[u_{1_{0}}\right]^{\alpha}}{2} e^{\lambda t}+\frac{\left[u_{2_{0}}\right]^{\alpha}+\left[u_{1_{0}}\right]^{\alpha}}{2} e^{-\lambda t} .}
\end{array}\right.
$$

Novamente, de acordo com o Teorema de representação, essas soluções são os $\alpha$-níveis da solução fuzzy $u(t)$.

Para o sistema dado por (3.6) definimos, em cada instante t, o diâmetro do intervalo $\left[\left[u_{1}(t)\right]^{\alpha} ;\left[u_{2}(t)\right]^{\alpha}\right]$, para os $\alpha$-níveis $0<\alpha \leq 1$, da seguinte forma:

$$
\operatorname{diam}\left[\left[u_{1}(t)\right]^{\alpha} ;\left[u_{2}(t)\right]^{\alpha}\right]=\left[u_{2}(t)\right]^{\alpha}-\left[u_{1}(t)\right]^{\alpha}, \quad 0<\alpha \leq 1, \quad t \in[0, T] .
$$

Com isso, podemos notar que os diâmetros, em cada $\alpha$-nível, são crescentes com o tempo. Isto pode ser interpretado como o aumento da incerteza com o passar do tempo, o que é bem razoável, de fato. 


\section{Método Numérico}

Para a obtenção das aproximações numéricas das soluções de cada equação dada no sistema (3.5), faremos uma discretização do intervalo [0,T], para um $\mathrm{T}$ prefixado, através de um conjunto $\left\{0=t_{0}<t_{1}<\ldots<t_{n}=T\right\}$; em seguida, construímos o esquema numérico para as aproximações seguindo o método de Euler, como sugerido em Ma, Friedman e Kandel [10], que se baseia nas aproximações de primeira ordem para $\left[u_{1}^{\prime}\right]^{\alpha}$ e $\left[u_{2}^{\prime}\right]^{\alpha}$ dadas pelas diferenças avançadas:

$$
\left[z^{\prime}\left(t_{i}\right)\right]^{\alpha} \approx \frac{\left[z\left(t_{i}+h\right)\right]^{\alpha}-\left[z\left(t_{i}\right)\right]^{\alpha}}{h} .
$$

Daí, chamando $z_{i}=z\left(t_{i}\right)$, com $t_{i}=t_{0}+i h$ para $1 \leq i \leq n$ e $h=\left(T-t_{0}\right) / n$ e usando (4.1) em (3.5), teremos:

$$
\begin{cases}{\left[u_{1_{i+1}}\right]^{\alpha}=\left[u_{1_{i}}\right]^{\alpha}-h \lambda\left[u_{2_{i}}\right]^{\alpha},} & 0<\alpha \leq 1, \\ {\left[u_{2_{i+1}}\right]^{\alpha}=\left[u_{2_{i}}\right]^{\alpha}-h \lambda\left[u_{1_{i}}\right]^{\alpha}} & 0<\alpha \leq 1 .\end{cases}
$$

Os códigos numéricos para as aproximações foram desenvolvidos através do MATLAB, onde incluímos os resultados numéricos para o método de Runge-Kutta de $4^{\underline{a}}$ ordem (para comparação), que estão apresentados através de gráficos, para melhor visualização. Nas figuras, apresentamos a solução numérica fuzzy $(\operatorname{Euler}(+)$ e Runge-Kutta $(*))$ e a solução analítica fuzzy descrita por (3.6).

\section{Resultados}

Para as simulações onde aplicamos o modelo, consideramos como condição inicial, para todos os materiais radioativos, uma massa "em torno de 100g", descrita pelo seguinte conjunto fuzzy:

$$
u_{0}(x)=\left\{\begin{array}{cl}
\frac{x-90}{10}, & \text { se } 90 \leq x<100, \\
\frac{110-x}{10}, & \text { se } 100 \leq x \leq 110, \\
0, & \text { caso contrário. }
\end{array} \quad \forall x \in \mathbb{R},\right.
$$

O gráfico da figura 1, abaixo, é o conjunto fuzzy dado pela equação (5.1), onde os pontos marcados representam os $\alpha$-níveis utilizados para iniciar as aproximações para os métodos numéricos.

Para a constante de desintegração $\lambda$, característica de cada elemento radioativo, utilizamos os valores apresentados em Bassanezi e Ferreira [3] , para os seguintes elementos:

- Chumbo $\left(P b^{210}\right): \lambda=0,315 \times 10^{-1} \mathrm{ano}^{-1}$;

- Rádio $\left(\mathrm{Ra}^{226}\right): \lambda=0,440 \times 10^{-3} \mathrm{ano}^{-1}$. 


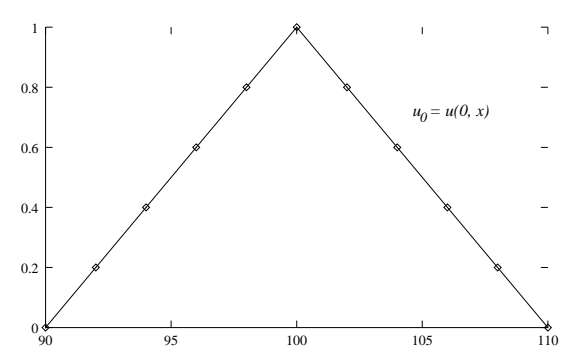

Figura 1: Condição inicial dada pela equação (5.1)

O intervalo de tempo considerado foi de zero a cento e vinte anos, com 60 iterações, ou seja, $h=2,0$ para $\left(P b^{210}\right)$. A seguir, apresentamos os gráficos das soluções analíticas e numéricas para o elemento $P b^{210}$ nas figuras 2 e 3 .

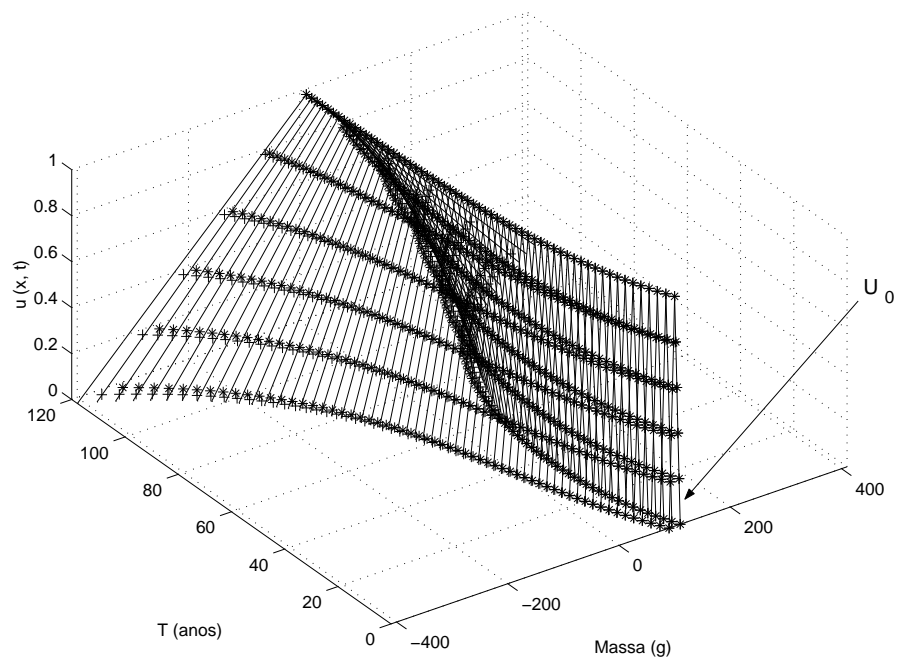

Figura 2: Gráfico do decaimento de $P b^{210}, u_{0}$ dado por $(5.1)$ e h $=2,0$.

Na figura abaixo, apresentamos o resultado obtido na última iteração, ou seja, em t $=120$ anos (figura 3-a). Para o cálculo do erro que aparece na figura 3b, chamando de " $u$ " a solução analítica, de "v" a solução numérica obtida pelo método de Euler e de " $y$ " a obtida por Runge-Kutta de $4^{\text {a }}$ ordem, definimos o erro da seguinte forma:

ErroEuler $=\operatorname{norm}(u-v)$;

ErroRunge-Kutta $_{\text {Rorm }}(u-y)$;

onde $(u-v)$ e $(u-y)$ são calculados ponto-a-ponto, em cada instante t, e "norm" é a norma euclidiana, dada por:

$$
\operatorname{norm}(\mathrm{x})=\sqrt{\sum_{k=1}^{N} x_{k}^{2}} .
$$






(a)



(b)

Figura 3: Comparação de resultados para $P b^{210}, u_{0}$ dado por $(5.1)$ e $\mathrm{h}=2,0$.

Para $R a^{226}$ obtivemos resultados mais satisfatórios, no sentido de melhor aproximação entre a solução analítica e as numéricas. Na figura 4, a seguir, utilizamos 100 iterações e $\mathrm{h}=30,0$.

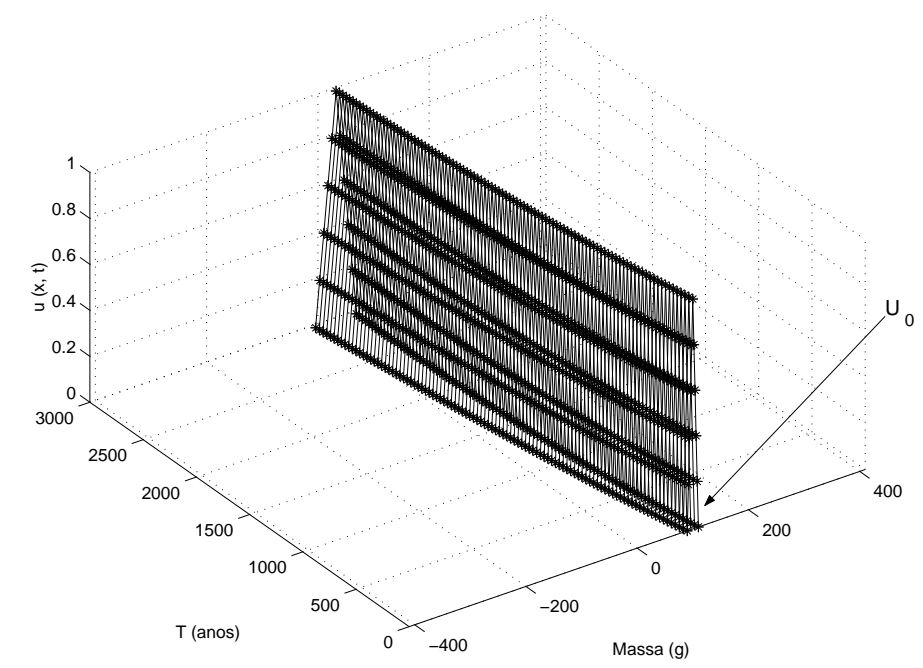

Figura 4: Gráfico do decaimento de $R a^{226}, u_{0}$ dado por $(5.1)$ e $\mathrm{h}=30,0$.

Os resultados apresentados nas figuras 5-a e 5-b, a seguir, mostram a boa apro- 
ximação obtida pelos dois métodos, cujos gráficos estão sobrepostos para o instante final $\mathrm{t}=3000$ anos, onde a solução analítica se justapõe às dadas por Euler $(+)$ e Runge-Kutta $(*)$.

O erro apresentado na figura 5 -b foi calculado da mesma forma, indicada anteriormente para a figura 3 -b.

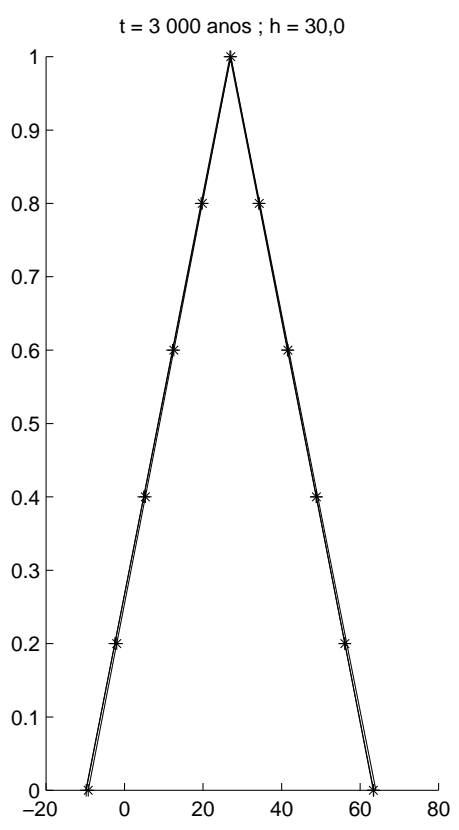

(a)

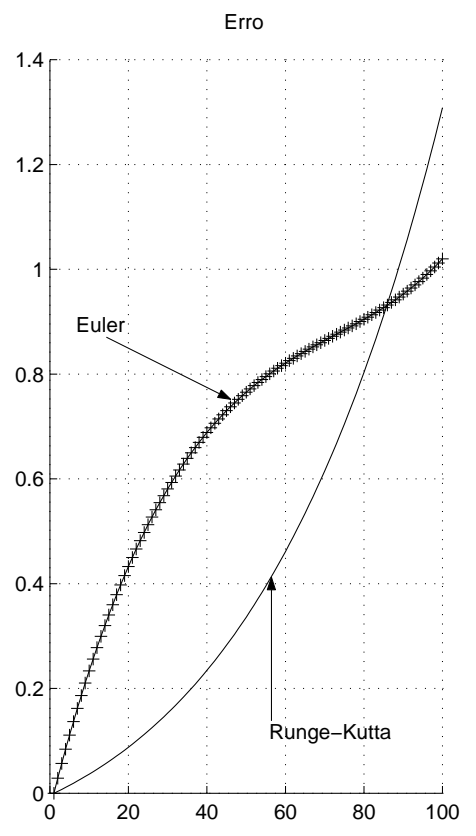

(b)

Figura 5: Comparação de resultados para $R a^{226}, u_{0}$ dado por $(5.1), \mathrm{h}=30,0$.

\section{Conclusões}

O método produziu aproximações bem mais satisfatórias entre a solução numérica fuzzy e a solução analítica fuzzy para $R a^{226}$ (figuras 4 e 5 ) do que para $P b^{210}$ (figuras 2 e 3), no sentido que, graficamente, estas soluções ficam cada vez mais diferentes. Mais precisamente, verificamos que a diferença entre os diâmetros dos intervalos da solução analítica fuzzy para as soluções numéricas fuzzy, em cada $\alpha$-nível, foi um pouco menor ao longo do tempo em $R a^{226}$ comparado a $P b^{210}$.

Apesar disso, foram compatíveis com a solução analítica fuzzy, uma vez que os diâmetros dos intervalos, para cada $\alpha$-nível, são crescentes com o tempo. Isto se explica pelo fato que, com o passar do tempo, aumenta a impossibilidade de afirmar precisamente o valor da emissão de radiação em cada instante. Este fato é, tecnicamente falando, uma conseqüência do conceito de taxa de variação fuzzy. Esta leva em conta as incertezas passadas e presentes para predizer o futuro, já que 
$u^{\prime}(t)=f(t, u(t))$ e $f$ é uma função fuzzy. O que, conforme mencionado antes, pode ser interpretado como aumento da incerteza com o passar do tempo.

A precisão do método numérico empregado (tipo Euler) é da ordem de h, para cada iteração. Mesmo assim, ele apresentou-se como um método confiável de aproximação da solução para escalas de tempo grande, como no caso do decaimento radioativo.

Para o $\alpha$-nível 1 ( $\alpha=1$ ), houve maior similaridade entre os resultados numéricos fuzzy e a solução analítica fuzzy. Porém, neste caso a solução fuzzy coincide com a solução determinística, apresentada no tratamento clássico do problema, dado pela equação (3.3), como provado em Barros et al [1].

Cabe observar, que para cada $t$ fixo a solução $u(t)$ mantém a forma inicial, ou seja, tipo triangular, o que geralmente não acontece para outros tipos de condição inicial para equações diferenciais fuzzy, como a considerada em (3.4).

Em futuros trabalhos, poderemos considerar o parâmetro $\lambda$ como um conjunto fuzzy (tipo triangular) de modo a melhorar o modelo, uma vez que o valor de $\lambda$ apresenta alguma incerteza também.

\begin{abstract}
The use of radioactive materials in the last century brought serious risk to the environment, mainly with reference to radioactive dumps. In this work, we have proposed a fuzzy model to describe the radioactive decay using fuzzy differential equations, the classic analytical solution of which is well known. We use the Euler and Runge-Kutta methods to obtain an approximate solution of an initial value problem of a fuzzy linear ordinary differential equation modelling radioactive decay. We compare the numerical results with the fuzzy analitical solution presented by Barros et al [2] for the similar fuzzy differential equation.
\end{abstract}

\title{
Referências
}

[1] L.C. Barros, R.C. Bassanezi e P.A. Tonelli, Remarks on deterministic orbits in fuzzy dynamical systems, em "Seventh IFSA World Congress", Vol. II, pp. 22-26, Prague, 1997.

[2] L.C. Barros, R.C. Bassanezi e P.A. Tonelli, Fuzzy modelling in population dynamics, Ecological Modelling 128 (2000), 27-33.

[3] R.C. Bassanezi e W.C. Ferreira Jr., "Equações Diferenciais com Aplicações", Ed. Harbra, São Paulo, 1978.

[4] S.L. Chang e L.A. Zadeh, On fuzzy mapping and Control, IEEE Trans. Systems Man Cybernetics 2 (1972), 30-34.

[5] D. Dubois e H. Prade, Towards fuzzy differential calculus: Part 3, differentiation, Fuzzy Sets and System 8 (1982), 225-233.

[6] O. Kaleva, Fuzzy differential equations, Fuzzy Sets and Systems 24 (1987), 301-317. 
[7] O. Kaleva, The Cauchy problem for fuzzy differential equations, Fuzzy Sets and Systems 35 (1990), 389-396.

[8] A. Kandel e W.J. Byatt, Fuzzy differential equations, em "Proc. Internat. Conf. Cybernetics and Society", pp. 1213-1216, Tóquio, 1981.

[9] A. Kandel e W.J. Byatt, Fuzzy process, Fuzzy Sets and Systems 4 (1980), 117-152.

[10] M. Ma, M. Friedmam e A. Kandel, Numerical solutions of fuzzy differential equations, Fuzzy Sets and Systems 105 (1999), 133-138.

[11] J.J. Nieto, The Cauchy problem for continuous fuzzy differential equations, Fuzzy Sets and Systems 102 (1999), 259-262.

[12] H. Ouyang e Y. Wu, On fuzzy differential equations, Fuzzy Sets and Systems 32 (1989), 321-325.

[13] M.L. Puri e D.A. Ralescu, Differentials of fuzzy functions, J. Math. Anal. Appl. 91 (1983), 552-558.

[14] S. Seikkala, On the fuzzy initial value problem, Fuzzy Sets and Systems 24 (1987), 319-330.

[15] C. Wu, S. Song e E.S. Lee, Approximate solutions, existence and uniqueness of the Cauchy problem of fuzzy differential equations, J. Math. Anal. Appl. 202 (1996), 629-644. 